\title{
FERTIRRIGAÇÃO NA PRODUÇÃo DE MUDAS DE Eucalyptus grandis NOS PERÍODOS DE INVERNO E VERÃO
}

\author{
Simone Fernandes Ciavatta ${ }^{1}$, Magali Ribeiro da Silva ${ }^{1 *}$, Danilo Simões ${ }^{1}$
}

*Autor para correspondência: magaliribeiro@fca.unesp.br

\begin{abstract}
RESUMO: Pesquisas correlacionando o parcelamento da fertirrigação com o desenvolvimento e qualidade de mudas florestais, em diferentes sistemas de produção e períodos do ano são fundamentais para a otimização de insumos e minimização do ciclo de produção. Dessa forma conduziu-se esse estudo com o objetivo de verificar a influência do parcelamento da fertirrigação no desenvolvimento e qualidade de mudas de Eucalyptus grandis nos períodos de inverno e verão. As fertilizações por meio do sistema de irrigação por capilaridade foram parceladas em: uma, duas, três ou seis vezes por semana, sendo mantida a quantidade total de nutrientes aplicados. Concluiu-se que, as fertirrigações mais eficazes para o desenvolvimento e a qualidade de mudas de Eucalyptus grandis foram de seis e uma vez na semana, para o período de inverno e verão, respectivamente, demonstrando-se a necessidade de adequação dos manejos de fertirrigação em função do período do ano.
\end{abstract}

Palavras-chave: viveiro florestal, adubação, eucalipto, parcelamento de fertilizantes.

\section{FERTIRRIGATION IN PRODUCTION OF SEEDLINGS OF Eucalyptus grandis DURING WINTER AND SUMMER}

\begin{abstract}
Studies correlating the splitting of fertigation with the development and quality of tree seedlings in different production systems and periods of the year are fundamental to optimize inputs and minimization of the production cycle. Thus we conducted this study aimed to check the influence of the split of fertigation in the development and quality of Eucalyptus grandis seedlings in winter and summer periods. The fertilization through the irrigation system by capillarity was split on: one, two, three or six times in the week, maintaining the total amount of nutrients applied. We concluded that the most effective fertigation for the development and quality of seedlings of Eucalyptus grandis were six and one times in the week for the period of winter and summer, respectively, demonstrating the need for adequacy of fertigation managements according to the period of year.
\end{abstract}

Keywords: forest nursery, fertilization, eucalypt, splitting of fertilizer.

\section{INTRODUÇÃO}

$\mathrm{Na}$ busca por mudas florestais de qualidade produzidas em um menor tempo possível, diversos insumos e manejos são usados no processo, o que determina resultados diferenciados, já que os fatores se interagem. Os manejos de irrigação e de fertilização devem ser adequados ao local em que o viveiro se encontra, à espécie, à época do ano, ao tipo de embalagem e ao substrato.

O manejo de fertilização nos viveiros florestais é alterado em função da fase de desenvolvimento das mudas e da época do ano. Quanto à fase, se classifica em adubação de crescimento e adubação de rustificação. A primeira visa o crescimento da muda e, portanto deve fornecer todos os nutrientes necessários para $o$ acúmulo de biomassa aérea e radicular, evitando que a muda apresente estresse nutricional. A segunda fase é caracterizada por adaptar a muda às condições de campo e, portanto pequenos estresses nutricionais podem fazem parte desta adaptação, já que normalmente se utiliza somente fertilizantes potássicos nesta fase. Quanto à época do ano Silveira et al. (2001) preconizam que a concentração da solução nutritiva no período de inverno deve ser cerca de $20 \%$ superior que a do verão.

Nos viveiros florestais de grande porte, a adubação geralmente por fertirrigação é a mais habitual, sendo uma técnica muito interessante para uso no processo de produção por possibilitar às mudas um desenvolvimento rápido e controlado (AUGUSTO et al., 2007) e o maior parcelamento dos nutrientes (COELHO et al., 2009). A eficiência da fertirrigação guarda estreita relação com o sistema de produção: irrigação, substrato, embalagem, fontes de adubo, bem como a época do ano e as características fisiológicas da espécie. Segundo Sgarbi et al. (1999) uma das alternativas para aumentar a eficiência das adubações é a realização de um maior parcelamento, principalmente quando se trata do nitrogênio.

1 Universidade Estadual Paulista "Júlio Mesquita Filho" - Botucatu, São Paulo, Brasil

Cerne, Lavras, v. 20, n. 2, p. 217-222, abr./jun. 2014 
Dessa forma, o aumento da frequência da fertilização, usando soluções com baixa concentração de nutrientes, é considerado uma das grandes vantagens do sistema de fertirrigação. Para Oliveira et al. (2007) esta técnica implica no uso racional de fertilizantes, já que aumenta a eficiência de seu uso, reduz mão-de-obra, além de flexibilizar a época de aplicação que compõem a operação. A maximização operacional e a minimização dos custos no processo de produção são de suma importância nas tomadas de decisões, o que as tornam mais viáveis economicamente (SIMÕES; SILVA, 2010).

Embora o conceito seja de que haja maior eficácia em manejos com maior frequência de fertilização utilizando soluções mais diluídas, são necessárias mais pesquisas correlacionando o parcelamento da fertirrigação, em diferentes sistemas de produção, com o desenvolvimento e qualidade de mudas florestais para que se possam fazer generalizações mais assertivas. Nesse contexto, este estudo visa verificar a influência do parcelamento da fertirrigação no desenvolvimento e na qualidade de mudas de Eucalyptus grandis, nos períodos de inverno e verão.

\section{MATERIAL E MÉTODOS}

O estudo foi realizado no Viveiro de Pesquisas de Mudas Florestais da Faculdade de Ciências Agronômicas da UNESP, campus de Botucatu, Estado de São Paulo, localizado nas coordenadas $22^{\circ} 51^{\prime} 22^{\prime \prime}$ de latitude Sul e $48^{\circ} 26^{\prime} 0$ " longitude Oeste, com altitude de $810 \mathrm{~m}$ e clima do tipo Cwa, segundo classificação de Wilhelm Köppen e precipitação média anual de $1.524 \mathrm{~mm}$. Os experimentos de inverno e de verão foram conduzidos, respectivamente, nos períodos de maio a agosto de 2008 e dezembro a março de 2009.

As sementes de Eucalyptus grandis (PCS-F1, com procedência de Santa Branca - SP) foram adquiridas do Instituto de Pesquisas e Estudos Florestais, Piracicaba-SP.

Foram utilizados tubetes com capacidade volumétrica de $50 \mathrm{~cm}^{3}$, preenchidos com substrato comercial a base de casca de pinus decomposta e vermiculita expandida. Como suportes para os tubetes foram usadas bandejas de polietileno tipo caixa com dimensões de $60 \mathrm{~cm}$ x $40 \mathrm{~cm}$, com capacidade para 96 mudas, porém com preenchimento de $50 \%$ das células.

A instalação do experimento deu-se com a preparação do substrato, sendo a este adicionado o correspondente a $300 \mathrm{~g}$ de FTE BR 12 (micronutrientes) por $\mathrm{m}^{3}$, homogeneizado em misturador; enchimento dos tubetes com substrato; irrigação e semeadura.

Cerne, Lavras, v. 20, n. 2, p. 217-222, abr./jun. 2014
As mudas permaneceram por aproximadamente 40 dias na casa de vegetação, sendo neste período realizado o raleio para deixar uma única muda por tubete. Após este período foram transferidas para casa de sombra, onde ficaram por 15 dias. Na sequência, foram levadas a uma casa de vegetação com cobertura plástica para controle da precipitação, permanecendo todo o período de aplicação das fertirrigações.

Para a irrigação, o sistema utilizado foi de microaspersão. Para a fertirrigação foi empregado o sistema de irrigação por capilaridade para garantir a homogeneidade de aplicação da solução nutritiva. Para esta finalidade construiu-se um tanque de alvenaria revestido com plástico transparente de uso agrícola, o qual era preenchido com as soluções nutritivas respectivas aos tratamentos, numa altura de $12 \mathrm{~cm}$, sendo esta $2 \mathrm{~cm}$ abaixo da borda superior do tubete. As bandejas de polietileno com as mudas permaneciam neste tanque até que fosse constatado o umedecimento da camada superficial do substrato (aproximadamente 8 minutos).

As fertilizações de crescimento e rustificação foram idênticas em relação à quantidade de adubo fornecida, porém diferentes na frequência de aplicação, sendo uma, duas, três ou seis vezes na semana. Dessa forma os experimentos (inverno e verão) foram compostos pelos tratamentos apresentados na Tabela 1.

Tabela 1 - Concentração de fertilizantes nas soluções nutritivas para os diferentes tratamentos.

Table 1 - Concentration of fertilizer in nutrient solutions for different treatments.

\begin{tabular}{ccccccc}
\hline & \multicolumn{9}{c}{ Fertilização de crescimento $\left(\mathrm{mg} \mathrm{L}^{-1}\right)$} & $\begin{array}{c}\text { Fertilização } \\
\text { de rustificação } \\
\text { Tratamentos }\end{array}$ \\
\cline { 2 - 6 } & $\mathrm{N}$ & $\mathrm{P}$ & $\mathrm{K}$ & $\mathrm{Ca}$ & $\mathrm{S}$ & $\mathrm{K}$ \\
\hline F1 & 1560,0 & 420,0 & 1080,0 & 1020,0 & 507,0 & 600,0 \\
F2 & 780,0 & 210,0 & 540,0 & 510,0 & 253,5 & 300,0 \\
F3 & 520,0 & 140,0 & 360,0 & 340,0 & 169,0 & 200,0 \\
F6 & 260,0 & 70,0 & 180,0 & 170,0 & 84,5 & 100,0 \\
\hline
\end{tabular}

${ }^{*}$ F1 - fertirrigação semanal; F2 - fertirrigação duas vezes na semana; - F3 - fertirrigação três vezes na semana; F6 - fertirrigação seis vezes na semana.

No experimento de inverno, a fertilização de crescimento iniciou-se aos 58 dias após a semeadura, permanecendo 34 dias e a fertilização de rustificação 
iniciou-se aos 94 dias, durante 21 dias. No experimento de verão, a fertilização de crescimento iniciou-se 58 dias após a semeadura e permaneceu por 30 dias, tendo a fertilização de rustificação iniciada aos 90 dias com 15 dias de duração.

Os fertilizantes utilizados na fase de crescimento das mudas foram: sulfato de amônio ( $20 \%$ de N e $24 \%$ de $\mathrm{S})$; monoamoniofosfato (MAP) purificado $\left(60 \%\right.$ de $\mathrm{P}_{2} \mathrm{O}_{5}$ e $12 \%$ de $\mathrm{N})$; nitrato de potássio (Krista $\left.\mathrm{K}^{\odot}\right)(45 \%$ de $\mathrm{K}_{2} \mathrm{O}$ e $12 \%$ de $\left.\mathrm{N}\right)$; nitrato de cálcio ( $15 \%$ de $\mathrm{N}$ e $20 \%$ de Ca). Para a fase de rustificação foi utilizado o cloreto de potássio $\left(60 \%\right.$ de $\left.\mathrm{K}_{2} \mathrm{O}\right)$.

As análises das soluções nutritivas foram realizadas semanalmente após cada troca de solução, medindo-se: condutividade elétrica (EC) e potencial hidrogeniônico $(\mathrm{pH})$, por meio de condutivímetro e de peagâmetro. Os valores médios encontrados estão apresentados na Tabela 2.

Tabela 2 - Valores médios de $\mathrm{pH}$ e EC $\left(\mathrm{dS} \mathrm{m}^{-1}\right)$ nas soluções nutritivas dos diferentes tratamentos durante a fase de crescimento e rustificação.

Table 2 - Average values of $\mathrm{pH}$ and EC $\left(\mathrm{dS} \mathrm{m}^{-1}\right)$ in nutrient solutions of different treatments during the growth and hardening phases.

\begin{tabular}{ccccc}
\hline \multirow{2}{*}{ Tratamentos } & \multicolumn{2}{c}{$\begin{array}{c}\text { Fertilização de } \\
\text { crescimento }\end{array}$} & \multicolumn{2}{c}{$\begin{array}{c}\text { Fertilização de } \\
\text { rustificação }\end{array}$} \\
\cline { 2 - 5 } & $\mathrm{pH}$ & $\mathrm{EC}\left(\mathrm{dS} \mathrm{m} \mathrm{m}^{-1}\right)$ & $\mathrm{pH}$ & $\mathrm{EC}\left(\mathrm{dS} \mathrm{m}{ }^{-1}\right)$ \\
\hline F1 & 5,7 & 7,2 & 7,6 & 1,8 \\
F2 & 5,8 & 4,3 & 7,3 & 1,2 \\
F3 & 6,0 & 2,6 & 7,3 & 0,6 \\
F6 & 6,2 & 1,6 & 7,6 & 0,4 \\
\hline
\end{tabular}

O delineamento experimental adotado foi inteiramente casualizado, composto por 4 tratamentos, constituídos por 5 parcelas de 48 plantas cada, sendo as 24 centrais consideradas úteis para as avaliações.

As avaliações morfológicas constaram de: altura da parte aérea (H), diâmetro de colo (DC), massa seca aérea (MSA), massa seca radicular (MSR), massa seca total (MST). Estes resultados foram submetidos à técnica da análise de variância. Para os efeitos significativos, foram realizados teste de média, pelo teste de Tukey a $5 \%$ de probabilidade.

A qualidade do sistema radicular (QR) foi avaliada conforme a metodologia utilizada por Silva et al. (2012), contudo caracterizadas da seguinte forma: "regular" sistema radicular estruturado, porém com alguma flexibilidade exigindo maior cuidado no momento do plantio no campo; "bom" sistema radicular estruturado, com torrão firme, porém sem presença de raízes brancas; "ótimo" sistema radicular totalmente estruturado com torrão firme e presença de raízes brancas, mostrando maior atividade fisiológica.

\section{RESULTADOS E DISCUSSÃO}

O desenvolvimento das mudas de Eucalyptus grandis no período de inverno (Tabela 3 ) foi influenciado pelo manejo de fertirrigação. De todas as características avaliadas, somente a massa seca aérea não diferiu estatisticamente entre os tratamentos. A MST foi estatisticamente inferior quando usada a solução nutritiva mais concentrada (F1). O IH foi menor estatisticamente nas soluções nutritivas mais concentradas (F1 e F2). O DC foi superior nas soluções nutritivas mais diluídas (F3 e F6) embora semelhante à F2, a qual não diferiu de F1. Quanto à MSR, F6 foi superior estatisticamente ao F1, os demais tratamentos ficaram com valores intermediários e semelhantes entre si.

Quanto ao padrão de desenvolvimento das mudas ao final do experimento, aos 115 dias após a semeadura, todos os tratamentos apresentaram alturas e diâmetros superiores aos sugeridos para plantio, ou seja, acima de $25 \mathrm{~cm}$ e 2,5 $\mathrm{mm}$, respectivamente, de acordo com Lopes (2004).

Tabela 3 - Caracterização morfológica das mudas de Eucalyptus grandis, aos 115 dias após semeadura, produzidas no inverno.

Table 3 - Morphological characterization of Eucalyptus grandis seedlings, 115 days after sowing, produced in winter.

\begin{tabular}{cccccc}
\hline Tratamentos & $\begin{array}{c}\text { IH } \\
(\mathrm{cm})\end{array}$ & $\begin{array}{c}\text { DC } \\
(\mathrm{mm})\end{array}$ & $\begin{array}{c}\text { MSA } \\
(\mathrm{g})\end{array}$ & $\begin{array}{c}\text { MSR } \\
(\mathrm{g})\end{array}$ & $\begin{array}{c}\text { MST } \\
(\mathrm{g})\end{array}$ \\
\hline F1 & $33,64 \mathrm{~b}$ & $3,03 \mathrm{~b}$ & $2,04 \mathrm{a}$ & $0,36 \mathrm{~b}$ & $2,40 \mathrm{~b}$ \\
F2 & $34,08 \mathrm{~b}$ & $3,19 \mathrm{ab}$ & $2,12 \mathrm{a}$ & $0,39 \mathrm{ab}$ & $2,51 \mathrm{a}$ \\
F3 & $36,74 \mathrm{a}$ & $3,28 \mathrm{a}$ & $2,15 \mathrm{a}$ & $0,42 \mathrm{ab}$ & $2,57 \mathrm{a}$ \\
F6 & $37,23 \mathrm{a}$ & $3,40 \mathrm{a}$ & $2,35 \mathrm{a}$ & $0,43 \mathrm{a}$ & $2,78 \mathrm{a}$ \\
\hline C.V. $(\%)$ & 10,90 & 11,34 & 26,24 & 29,05 & 24,22 \\
\hline
\end{tabular}

- médias seguidas de letras iguais na mesma coluna não diferem pelo Teste de Tukey, ao nível de 5\% de significância.

- $\mathrm{IH}=$ incremento em altura; $\mathrm{D}=$ diâmetro de colo; $\mathrm{MSA}=$ massa seca da parte aérea; $\mathrm{MSR}=$ massa seca da parte radicular; $\mathrm{MST}=$ massa seca total; $\mathrm{CV}=$ coeficiente de variação.

Cerne, Lavras, v. 20, n. 2, p. 217-222, abr./jun. 2014 
Segundo Augusto et al. (2007), estudando a produção de mudas de Eucalyptus grandis via seminal e usando o sistema de irrigação por capilaridade, porém com dois tipos de solução, água residuária sem fertilização e água potável com adição de fertilizantes (sistema convencional), verificaram que o desenvolvimento das mudas foi superior no sistema convencional, provavelmente pela maior concentração de macronutrientes na solução nutritiva deste sistema. Também no estudo feito por Sarzi et al. (2008b) verificaram que em mudas de Tabebuia chrysotricha em substrato contendo $100 \%$ fibra de coco granulada, com fertirrigações semanais via capilaridade, as maiores concentrações de sais proporcionaram maiores médias de altura e massa seca.

O fato do menor desenvolvimento das mudas, durante o inverno, ter sido verificado no tratamento de menor freqüência com solução mais concentrada, contradiz estudos que recomendam uma solução nutritiva mais concentrada na época de inverno (HIGASHI; SILVEIRA, 2004; SILVEIRA et al., 2001), pois esperavase que as soluções mais concentradas, nesta época do ano, resultassem em maiores desenvolvimentos das mudas, já que com a baixa evapotranspiração deste período, a absorção é reduzida exigindo uma solução nutritiva mais concentrada para suprir a demanda das plantas. Provavelmente, esse resultado deve-se ao fato da aplicação da solução nutritiva ser via irrigação por capilaridade, um sistema mais eficiente na absorção da solução.

Ao avaliar a qualidade do sistema radicular (Tabela 4) observou-se que as mudas produzidas com maior frequência de fertirrigação (F6), apresentaram sistemas radiculares melhores, com $70,0 \%$ caracterizado como ótimo, $28,0 \%$ como bom e somente $2,0 \%$ de mudas consideradas regulares, que exigem maior cuidado durante o plantio em função do torrão não estar totalmente firme. Os demais tratamentos apresentaram mudas com sistemas radiculares inferiores, variando de 42,0 a $52,0 \%$ com qualidade ótima, 37,5 a $44,0 \%$ boa e de 8,0 a $14,0 \%$ regular. Davis e Jacobs (2005) relatam que a morfologia do sistema radicular e o estado fisiológico pode fornecer uma indicação mais precisa do potencial das mudas, no entanto, poucos estudos têm tentado avaliar quantitativamente a qualidade do sistema radicular e sua relação com o sucesso do plantio. Freitas et al. (2005) também mencionam que a quantidade de raízes finas no sistema radicular é um dos fatores que podem interferir no desempenho inicial das mudas no campo, uma vez que mudas que apresentam grande produção dessas raízes são mais aptas a condições de estresse ambiental, garantindo maiores taxas de sobrevivência e crescimento inicial após o plantio.

Tabela 4 - Qualidade do sistema radicular das mudas de Eucalyptus grandis, aos 115 dias após semeadura, produzidas no inverno.

Table 4 - Quality of root system of Eucalyptus grandis seedlings, 115 days after sowing, produced in winter.

\begin{tabular}{cccccc}
\hline \multirow{2}{*}{ Tratamentos } & \multicolumn{2}{c}{$\begin{array}{c}\text { Qualidade do sistema } \\
\text { radicular (\%) }\end{array}$} & $\begin{array}{c}\text { Aptos p/ } \\
\text { plantio (\%) }\end{array}$ & $\begin{array}{c}\text { Aptos p/ } \\
\text { plantio, com } \\
\text { restrições (\%) }\end{array}$ \\
\cline { 2 - 6 } & Ótimo & Bom Regular & & 8,0 \\
\hline F1 & 52,0 & 40,0 & 8,0 & 92,0 & 14,0 \\
F2 & 42,0 & 44,0 & 14,0 & 86,0 & 12,5 \\
F3 & 50,0 & 37,5 & 12,5 & 87,5 & 2,0 \\
F6 & 70,0 & 28,0 & 2,0 & 98,0 & 2,0 \\
\hline
\end{tabular}

No verão, a solução nutritiva mais concentrada (F1) produziu mudas com MSA e MST superiores estatisticamente às demais soluções avaliadas e com $\mathrm{IH}$ semelhante àquela que utilizou a solução mais diluída (F6). As características DC e MSR não foram influenciadas pelos manejos de fertirrigação (Tabela 5).

Sarzi et al. (2008a) verificaram que as soluções de fertirrigação com EC de 1,06; 2,12; 3,2 e 4,24 dS m ${ }^{-1}$ influenciaram pouco no desenvolvimento de mudas de ipêamarelo-cascudo em altura, diâmetro de colo, número de pares de folhas e matéria seca total. De acordo com dados de Gomes et al. (2003), a altura das mudas adequados para plantio está entre 20 a $35 \mathrm{~cm}$. Quanto ao diâmetro, os valores obtidos foram superiores aos de Lopes (2004) e Sturion et al. (2000) os quais sugerem valores acima de 2,0 e 2,5 , respectivamente.

De forma semelhante ao experimento de inverno, o resultado esperado para o experimento de verão, no que se refere ao desenvolvimento da muda não corroborou com o manejo preconizado pelos viveiros florestais, ou seja, fertilizações mais frequentes com soluções mais diluídas para o verão.

Quanto à qualidade do sistema radicular (Tabela 6) observou-se que as mudas produzidas com as frequencias de fertirrigações F1 e F6 foram semelhantes entre si, indicando que a qualidade do sistema radicular não foi influenciada quando utilizadas soluções nutritivas com maior e menor concentração, no período de verão. De acordo com Novaes et al. (2002), alguns pesquisadores

Cerne, Lavras, v. 20, n. 2, p. 217-222, abr./jun. 2014 
têm ressaltado a importância das raízes no desempenho das plantas após o plantio, dadas às atividades fisiológicas das quais dependem o crescimento das plantas.

Tabela 5 - Caracterização morfológica das mudas de Eucalyptus grandis, aos 105 dias após semeadura, produzidas no verão.

Table 5 - Morphological characterization of Eucalyptus grandis seedlings, 105 days after sowing, produced in summer.

\begin{tabular}{cccccc}
\hline Tratamentos & $\begin{array}{c}\text { IH } \\
(\mathrm{cm})\end{array}$ & $\begin{array}{c}\text { DC } \\
(\mathrm{mm})\end{array}$ & $\begin{array}{c}\text { MSA } \\
(\mathrm{g})\end{array}$ & $\begin{array}{c}\text { MSR } \\
(\mathrm{g})\end{array}$ & $\begin{array}{c}\text { MST } \\
(\mathrm{g})\end{array}$ \\
\hline F1 & $34,8 \mathrm{ab}$ & $4,11 \mathrm{a}$ & $3,22 \mathrm{a}$ & $0,62 \mathrm{a}$ & $3,84 \mathrm{a}$ \\
F2 & $33,5 \mathrm{~b}$ & $3,98 \mathrm{a}$ & $2,46 \mathrm{~b}$ & $0,57 \mathrm{a}$ & $3,03 \mathrm{~b}$ \\
F3 & $30,6 \mathrm{c}$ & $4,03 \mathrm{a}$ & $2,45 \mathrm{~b}$ & $0,61 \mathrm{a}$ & $3,06 \mathrm{~b}$ \\
F6 & $36,4 \mathrm{a}$ & $4,03 \mathrm{a}$ & $2,66 \mathrm{~b}$ & $0,56 \mathrm{a}$ & $3,22 \mathrm{~b}$ \\
CV(\%) & 17,13 & 10,39 & 22,70 & 21,44 & 20,93 \\
\hline
\end{tabular}

Médias seguidas de letras iguais na mesma coluna não diferem pelo teste de Tukey, ao nível de $5 \%$ de significância. $\mathrm{IH}=$ incremento em altura; $\mathrm{D}=$ diâmetro de colo; $\mathrm{MSA}=$ massa seca da parte aérea; $\mathrm{MSR}=$ massa seca da parte radicular; $\mathrm{MST}=$ massa seca total; $\mathrm{CV}=$ coeficiente de variação

Tabela 6 - Qualidade do sistema radicular das mudas de Eucalyptus grandis, aos 105 dias após semeadura, produzidas no verão.

Table 6 - Quality of root system of Eucalyptus grandis seedlings, 105 days after sowing, produced in summer.

\begin{tabular}{cccccc}
\hline \multirow{2}{*}{ Tratamentos } & \multicolumn{2}{c}{$\begin{array}{c}\text { Qualidade do sistema } \\
\text { radicular (\%) }\end{array}$} & $\begin{array}{c}\text { Aptos p/ } \\
\text { plantio } \\
\text { (\%) }\end{array}$ & $\begin{array}{c}\text { Aptos p/ } \\
\text { Olantio, com } \\
\text { Ótimo }\end{array}$ & $\begin{array}{c}\text { Bom } \\
\text { restrições } \\
(\%)\end{array}$ \\
\hline F1 & 37,5 & 45,0 & 17,5 & 82,5 & 17,5 \\
F2 & 32,5 & 42,5 & 25,0 & 75,0 & 25,0 \\
F3 & 37,5 & 52,5 & 10,0 & 90,0 & 10,0 \\
F6 & 42,5 & 40,0 & 17,5 & 82,5 & 17,5 \\
\hline
\end{tabular}

\section{CONCLUSÕES}

O parcelamento da fertirrigação interferiu no desenvolvimento e qualidade de mudas de Eucalyptus grandis de forma diferente nos períodos de inverno e verão.

A fertirrigação com solução nutritiva mais diluída e em maior frequência de aplicação foi o manejo mais eficaz para o período de inverno. Para o período de verão, a solução mais concentrada aplicada uma única vez na semana, caracterizou-se como o melhor manejo a ser adotado.

\section{AGRADECIMENTOS}

À Coordenação de Aperfeiçoamento de Pessoal de Nível Superior - CAPES, pela concessão de bolsa de estudos.

\section{REFERÊNCIAS}

AUGUSTO, D. C. C.; GUERRINI, I. A.; ENGEL, V. L.; ROUSSEAU, G. X. Utilização de águas residuárias provenientes do tratamento biológico de esgotos domésticos na produção de mudas de Eucalyptus grandis Hill. Ex. Maiden. Revista Árvore, Viçosa, v. 31, n. 4, p. 745-751, jul./ago. 2007.

COELHO, G.; SILVA, A. M.; REZENDE, F. C.; SILVA, R. A.; CUSTÓDIO, A. A. PAIVA. Efeito de épocas de irrigação e de parcelamento de adubação sobre a produtividade do cafeeiro "catuaí". Ciência e Agrotecnologia, Lavras, v. 33, n. 1, p. 67-78, jan./fev. 2009.

DAVIS, A. S.; JACOBS, D. F. Quantifying root system quality of nursery seedlings and relationship to outoplanting performance. New Forests, Melbourne, n. 30, p. 295-311, 2005.

FREITAS, T. A. S. de; BARROSO, D.; CARNEIRO, J. G. de A.; PENCHEL, R. M.; LAMÔNICA, K. R.; FERREIRA, D. de A.Desempenho radicular de mudas de eucalipto produzidas em diferentes recipientes e substratos. Revista Árvore, Viçosa, v.29, n.6, p.853861, nov./dez. 2005.

GOMES, J. M.; COUTO, L.; LEITE, H. G.; XAVIER, A.; GARCIA, S. L. R. G. Crescimento de mudas de Eucalyptus grandis em diferentes tamanhos de tubetes e fertilização N-P-K. Revista Árvore, Viçosa, v. 27, n. 2, p. 113-127, mar./abr. 2003.

HIGASHI, E. N.; SILVEIRA, R. L. A. Fertirrigação em viveiros de mudas de Eucalyptus e Pinus. In: BOARETTO, A. E.; VILLAS-BOAS, R. L.; SOUZA, W. F. PARRAS, L. R. V. (Ed.). Fertirrigação: teoria e prática. Piracicaba: FEALQ, 2004. v. 1, p. 677-725. 1 CD-ROM.

LOPES, J. L. W. Produção de mudas de Eucalyptus grandis W. (Hill ex. Maiden) em diferentes substratos e lâminas de irrigação. 2004. 100 p. Dissertação (Mestrado em Irrigação e Drenagem) - Universidade Estadual Paulista, Botucatu, 2004.

Cerne, Lavras, v. 20, n. 2, p. 217-222, abr./jun. 2014 
NOVAES, A. B.; CARNEIRO, J. G. A.; BARROSOS, D. G.; LELES, P. S. S. Avaliação do potencial de regeneração de raízes de mudas de Pinus taeda L., produzidas em diferentes tipos de recipientes, e o seu desempenho no campo. Revista Árvore, Viçosa, v. 26, n. 6, p. 675-681, nov./dez. 2002.

OLIVEIRA, S. L.; COELHO, E.F; BORGES, A. L. Banana produção: aspectos técnicos. Brasília: Embrapa, 2000, 143p.

SARZI, I.; VILLAS-BOAS, R. L.; SILVA, M. R. da. Desenvolvimento de mudas de Tabebuia chrysotricha em função de substratos e de soluções de fertirrigação.

Cerne, Lavras, v. 14, n. 2, p. 153-162, abr./jun. 2008a.

SARZI, I.; VILLAS-BOAS, R. L.; SILVA, M. R. da. Composição química e aspectos morfológicos de mudas de Tabeuia chrysotricha (Standl.) produzidas em diferentes substratos e soluções de fertirrigação. Revista Scientia Forestalis, Piracicaba, v. 36, n. 77, p. 53-62, mar. 2008b.

SGARBI, F.; SILVEIRA, R. L. V. A.; TAKAHASHI, E. N.; CAMARGO, M. A. F. Crescimento e produção de biomassa de clone de Eucalyptus grandis $\mathrm{x}$ Eucalyptus urophylla em condições de deficiência de macronutrientes, B e Zn. Revista Scientia Forestalis, Piracicaba, n. 56, p. 69-82, dez. 1999.

SILVA, R. B. G.; SIMÕES, D.; SILVA, M. R. Qualidade de mudas clonais de Eucalyptus urophylla x $E$. grandis em função do substrato. Revista Brasileira de Engenharia Agrícola e Ambiental, Campina Grande, v. 16, n. 3, p. 297-302, mar. 2012.

SILVEIRA, R. L. V. A.; HIGASHI, E. N.; SGARBI, F.; MUNIZ, M. R. A. Seja o doutor do seu eucalipto. Piracicaba: POTAFÓS, 2001. 32 p.

SIMÕES, D.; SILVA, M. R. da. Análise técnica e econômica das etapas de produção de mudas de eucalipto. Cerne, Lavras, v. 16, n. 3, p. 359-366, jul./set. 2010.

STURION, J. A.; GRACA, L. R.; ANTUNES, J. B. $M$. Produção de mudas de espécies de rápido por pequenos produtores. Colombo: EMBRAPA florestas, 2000. 21 p. (Circular Técnica, 37).

Recebido: 17 de outubro de 2011; aceito: 23 de agosto de 2013.

Cerne, Lavras, v. 20, n. 2, p. 217-222, abr./jun. 2014 